\title{
Excitation Mechanisms in Seyfert Galaxies
}

\author{
M. G. Allen and M. A. Dopita
}

Mt. Stromlo and Siding Spring Observatories, Private Bag, Weston Creek P.O., ACT 2611, Australia

\section{Z. I. Tsvetanov}

Department of Physics and Astronomy, The Johns Hopkins University, Baltimore, MD 21218, USA

\section{Introduction}

The excitation mechanism of the extended emission-line regions (EELRs) in Seyfert galaxies is commonly thought to be nuclear photoionization. However, some Seyfert galaxies have EELRs with disturbed kinematics, outflows, and morphology which is indicative of interaction between radio plasma and the ISM of the host galaxy. In these cases shock excitation must also be considered (Bicknell 1993).

To investigate the importance of shocks and photoionization, we have taken two approaches: mapping the spatial dependence of the line ratios, and using UV line ratios which are sensitive discriminants between shocks and photoionization.

\section{Models and Observations}

Shock and photoionization models were calculated with the MAPPINGS II code. The shock models are steady 1-d radiative flows (Dopita \& Sutherland 1995) with shock velocities $150-500 \mathrm{~km} \mathrm{~s}^{-1}$ and magnetic parameter $B n^{-1 / 2}=0-$ $4 \mu \mathrm{G} \mathrm{cm}^{3 / 2}$. The photoionization models use a power law $\left(F_{\nu} \propto \nu^{\alpha}\right)$, with a high-energy cutoff at $100 \mathrm{Ryd}, n_{e}=100-1000 \mathrm{~cm}^{-3}$, spectral indices $\alpha=-1$, -1.4 , and -2 , ionization parameter $U=0.0001-1.0$, and are ionization bounded.

While shock and photoionization models predict similar optical spectra, the predictions for the ratios of the UV lines C IV, C III], C1I, and He 11 differ significantly. This provides us with a means to discriminate between the models.

Observations include long-slit spectral maps $(3000-10000 \AA)$ of the EELRs in several prominent Seyfert galaxies taken with the ANU 2.3-m telescope at Siding Spring, and HST FOS spectra of the brightest position in the NGC 5728 and NGC 5643 EELRs and in the disk of M 87. Ground-based spectra of NGC 5728 and NGC 5643 were scaled to match the flux in the $[\mathrm{O} I I I]+\mathrm{H} \beta$ complex in the FOS prism spectrum to give a combined wavelength range of $1150-10000 \AA$. 

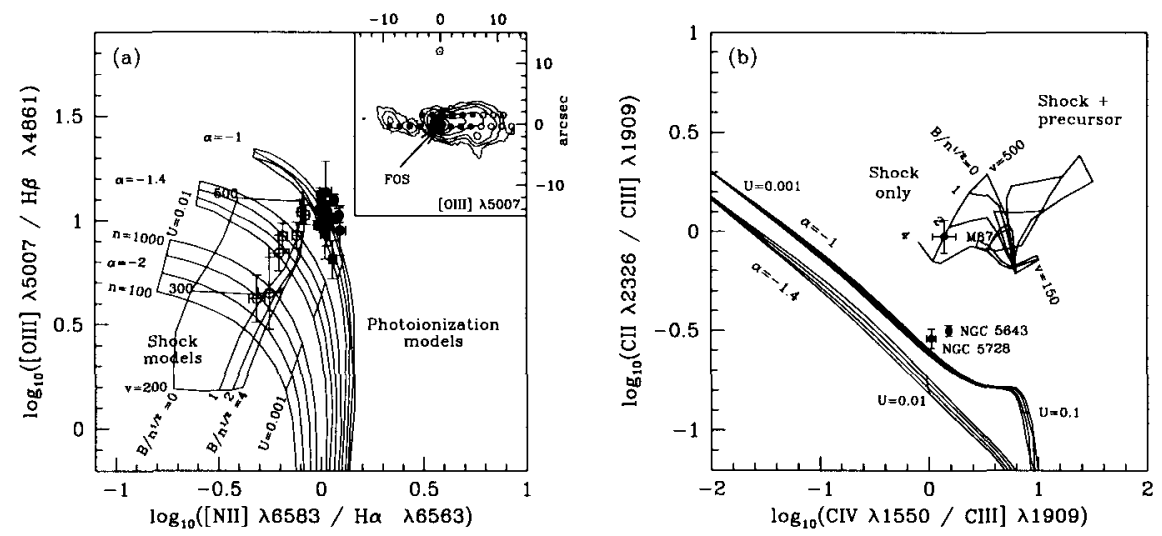

Figure 1. (a) NGC 5643 optical line ratios and model grids. (b) UV diagnostic diagram measurements for three galaxies.

\section{Results}

The optical spectral maps reveal strong spatial dependence of the line ratios which results in clear separations on diagnostic diagrams. Our sample of six EELRs show a range of spatial dependences, including different $[\mathrm{N} \mathrm{II}] / \mathrm{H} \alpha$ on either side of bipolar EELRs, lower-excitation spectra at the edges, and individual knots with different line ratios. Figure 1a, for example, shows the variation of $\left[\mathrm{O}_{\mathrm{III}}\right] / \mathrm{H} \beta$ and $[\mathrm{N} \mathrm{II}] / \mathrm{H} \alpha$ in NGC 5643 . The (open/solid) points correspond to spectra at the location of the (open/solid) points over-plotted on the [O III] image contours in the inset. The location of the FOS observation is indicated.

The FOS UV observations are plotted on the $\mathrm{C}_{\text {II }} / \mathrm{CIII}_{\mathrm{II}}$ vs. C IV/C III] diagram in Fig. 1b. (Note that shock and photoionization models are separated with this combination of line ratios). The measurements of NGC 5643 and NGC 5728 are closer to the photoionization models, but require higher ionization parameter $(U \approx 0.01)$ than indicated by the optical lines $(U \approx 0.003-0.005)$. Line-ratio measurements in the disk of M 87 fall within the shock-only grids.

Pending a complete analysis to be presented elsewhere we summarize: (a) the variation of the optical line ratios with respect to the model grids indicate that that there is more than one excitation mechanism in the EELR; and (b) the intensity peaks of the EELR in NGC 5643 and NGC 5728 are likely photoionized, and the disk of M 87 has shock-excited emission lines.

\section{References}

Bicknell, G.V. 1993, in The Physics of Active Galactic Nuclei, ed. Bicknell, Dopita, \& Quinn, ASP Conf. Ser. 54, p. 231.

Dopita, M. A., \& Sutherland, R. S. 1995, ApJ, 455, 468. 